International Electronic Journal of Geometry

Volume 7 No. 1 Pp. 154-162 (2014) @)IEJG

\title{
A NEW KIND OF HELICOIDAL SURFACE OF VALUE $M$
}

\author{
ERHAN GÜLER
}

Dedicated to memory of Professor Franki Dillen

\begin{abstract}
We define a new kind of helicoidal surface of value $m$. A rotational surface which is isometric to the helicoidal surface of value $m$ is revealed. In addition, we calculate some differential geometric properties of the helicoidal surface of value 3 in three dimensional Euclidean space.
\end{abstract}

\section{INTRODUCTION.}

In classical surface geometry, the right helicoid (resp. catenoid) is the only ruled (resp. rotational) surface which is minimal in Euclidean space. If we focus on the ruled (helicoid) and rotational characters, we have Bour's theorem in [4]. The French Mathematician Edmond Bour used semi-geodesic coordinates and found a number of new cases of the deformation of surfaces in 1862 . He also gave a well known theorem about the helicoidal and rotational surfaces.

Kenmotsu, [14] focuses on the surfaces of revolution with prescribed mean curvature. About helicoidal surfaces in Euclidean 3-space, do Carmo and Dajczer [5] prove that, by using a result of Bour [4], there exists a two-parameter family of helicoidal surfaces isometric to a given helicoidal surface. By making use of this parametrization, they found a representation formula for helicoidal surfaces with constant mean curvature. Furthermore they prove that the associated family of Delaunay surfaces is made up by helicoidal surfaces of constant mean curvature. Hitt and Roussos [11] also study on the helicoidal surfaces with constant mean curvature using computer graphics. Baikoussis and Koufogiorgos [1] prove that the helicoidal surfaces satisfying $K_{I I}=H$ are locally characterized by the constancy of the ratio of the principal curvatures. Ikawa determines pairs of surfaces by Bour's theorem with the additional condition that they have the same Gauss map in Euclidean 3-space in [12]. Some relations among the Laplace-Beltrami operator and curvatures of the helicoidal surfaces in Euclidean 3-space are shown by Güler et al in [9]. They give Bour's theorem on the Gauss map, and some special examples.

On the other hand, Dillen and Sodsiri [6] study ruled linear Weingarten surfaces in Minkowski 3-space. See also Minkowskian cases in ([2, 3, 8, 9, 13]).

2010 Mathematics Subject Classification. Primary 53C50; Secondary 53C40.

Key words and phrases. Helicoidal surface of value $m$, rotational surface of value $m$, mean curvature, Gaussian curvature, Gauss map. 
In section 2, we recall some basic notions of the Euclidean geometry, and the reader can be found the definitions of helicoidal and rotational surfaces of value $m$. Isometric helicoidal and rotational surfaces of value $m$ are obtained by Bour's theorem in section 3. Finally, isometric helicoidal and rotational surfaces of value 3 are examined in the last section.

\section{Preliminaries}

We shall identify a vector $(a, b, c)$ with its transpose. In this section, we will obtain the rotational and helicoidal surfaces in Euclidean 3-space. The reader can be found basic elements of differential geometry in $[4,7,15,16]$.

Now we define the rotational surface and helicoidal surface in $\mathbb{E}^{3}$. For an open interval $I \subset \mathbb{R}$, let $\gamma: I \longrightarrow \Pi$ be a curve in a plane $\Pi$ in $\mathbb{E}^{3}$, and let $\ell$ be a straight line in $\Pi$. A rotational surface in $\mathbb{E}^{3}$ is defined as a surface rotating a curve $\gamma$ around a line $\ell$ (these are called the profile curve and the axis, respectively). Suppose that when a profile curve $\gamma$ rotates around the axis $\ell$, it simultaneously displaces parallel lines orthogonal to the axis $\ell$, so that the speed of displacement is proportional to the speed of rotation. Then the resulting surface is called the helicoidal surface with axis $\ell$ and pitch $a \in \mathbb{R}^{+}$.

We may suppose that $\ell$ is the line spanned by the vector $(0,0,1)$. The orthogonal matrix which fixes the above vector is

$$
A(\theta)=\left(\begin{array}{ccc}
\cos \theta & -\sin \theta & 0 \\
\sin \theta & \cos \theta & 0 \\
0 & 0 & 1
\end{array}\right), \quad \theta \in \mathbb{R} .
$$

The matrix $A$ can be found by solving the following equations simultaneously; $A \ell=\ell, A^{t} A=A A^{t}=I_{3}$, $\operatorname{det} A=1$. When the axis of rotation is $\ell$, there is an Euclidean transformation by which the axis is $\ell$ transformed to the $z$-axis of $\mathbb{E}^{3}$. Parametrization of the profile curve is given by $\gamma(r)=(r, 0, \varphi(r))$, where $\varphi(r): I \subset \mathbb{R} \longrightarrow \mathbb{R}$ are differentiable function for all $r \in I$. A helicoidal surface in three dimensional Euclidean space which is spanned by the vector $(0,0,1)$ with pitch $a$, as follow

$$
\mathbf{H}(r, \theta)=A(\theta) \cdot \gamma(r)+a \theta \ell .
$$

When $a=0$, helicoidal surface is just a rotational surface.

\section{Helicoidal surfaces of value $m$}

We define a new kind of helicoidal surface, and using Bour's theorem we reveal a kind of isometric rotational surface in this section.

A helicoidal surface of value $m$ is defined by

$$
\mathbf{H}_{m}(r, \theta)=\mathbf{H}_{m}^{1}(r, \theta)+\mathbf{H}_{m}^{2}(r, \theta),
$$

where $\mathbf{H}_{m}^{1}(r, \theta)=\Re_{m}^{1} \cdot \gamma_{m}^{1}+\frac{1}{2} a \theta \ell, \mathbf{H}_{m}^{2}(r, \theta)=\Re_{m}^{2} \cdot \gamma_{m}^{2}+\frac{1}{2} a \theta \ell$, rotating matrices $\Re_{m}^{1}$ and $\Re_{m}^{2}$ are

$$
\Re_{m}^{1}(\theta)=\left(\begin{array}{ccc}
\cos [(m-1) \theta] & \sin [(m-1) \theta] & 0 \\
-\sin [(m-1) \theta] & \cos [(m-1) \theta] & 0 \\
0 & 0 & 1
\end{array}\right)
$$


and

$$
\Re_{m}^{2}(\theta)=\left(\begin{array}{ccc}
\cos [(m+1) \theta] & -\sin [(m+1) \theta] & 0 \\
\sin [(m+1) \theta] & \cos [(m+1) \theta] & 0 \\
0 & 0 & 1
\end{array}\right) .
$$

$\ell=(0,0,1)$ is the rotating axis, and the profile curves are

$$
\gamma_{m}^{1}(r)=\left(\frac{r^{m-1}}{m-1}, 0, \frac{1}{2} \varphi(r)\right), \gamma_{m}^{2}(r)=\left(-\frac{r^{m+1}}{m+1}, 0, \frac{1}{2} \varphi(r)\right),
$$

$m \in \mathbb{R}-\{1\}$ in $\gamma_{m}^{1}, m \in \mathbb{R}-\{-1\}$ in $\gamma_{m}^{2}, r \in \mathbb{R}^{+}, 0 \leq \theta \leq 2 \pi$, and the pitch $a \in \mathbb{R}^{+}$. Since the helicoidal surface is given by rotating the profile curves $\gamma$ around the axis $\ell$ and simultaneously displacing parallel lines orthogonal to the axis $\ell$, so that the speed of displacement is proportional to the speed of rotation. So, we have the following representation of the helicoidal surface of value $m$ in the following theorem.

Theorem 3.1. A helicoidal surface of value $m$ (in (3.1) is reduces to)

$$
\mathbf{H}_{m}(r, \theta)=\left(\begin{array}{c}
\frac{r^{m-1}}{m-1} \cos [(m-1) \theta]-\frac{r^{m+1}}{m+1} \cos [(m+1) \theta] \\
-\frac{r^{m-1}}{m-1} \sin [(m-1) \theta]-\frac{r^{m+1}}{m+1} \sin [(m+1) \theta] \\
\varphi(r)+a \theta
\end{array}\right),
$$

is isometric to the rotational surface of value $m$

$$
\mathbf{R}_{m}\left(r_{\mathbf{R}}, \theta_{\mathbf{R}}\right)=\left(\begin{array}{c}
\frac{r_{\mathbf{R}}^{m-1}}{m_{-1}} \cos \left[(m-1) \theta_{\mathbf{R}}\right]-\frac{r_{\mathbf{R}}^{m+1}}{m_{+1}} \cos \left[(m+1) \theta_{\mathbf{R}}\right] \\
-\frac{r_{\mathbf{R}}^{m-1}}{m-1} \sin \left[(m-1) \theta_{\mathbf{R}}\right]-\frac{r_{\mathbf{R}}^{m+1}}{m+1} \sin \left[(m+1) \theta_{\mathbf{R}}\right] \\
\varphi_{\mathbf{R}}\left(r_{\mathbf{R}}\right)
\end{array}\right)
$$

by Bour's theorem, where

$$
\begin{aligned}
\varphi_{\mathbf{R}}^{\prime 2}= & \frac{\left[2(m+1) r_{\mathbf{R}}^{2 m+1}+4 m r_{\mathbf{R}}^{2 m-1} \cos \left(2 m \theta_{\mathbf{R}}\right)\right]^{2} \operatorname{det} I}{\left[2(m+1) r^{2 m+1}+4 m r^{2 m-1} \cos (2 m \theta)\right]^{2} G} \\
& +\frac{2 r_{\mathbf{R}}^{2 m} \sin ^{2}\left(2 m \theta_{\mathbf{R}}\right)}{r_{\mathbf{R}}^{4}+2 r_{\mathbf{R}}^{2} \cos \left(2 m \theta_{\mathbf{R}}\right)+1} \\
& -r_{\mathbf{R}}^{2 m-4}\left(r_{\mathbf{R}}^{4}-2 r_{\mathbf{R}}^{2} \cos \left(2 m \theta_{\mathbf{R}}\right)+1\right), \\
r_{\mathbf{R}} & =\sqrt{G}, \\
\theta_{\mathbf{R}} & =\theta+\int \frac{F}{G} d r, \\
E & =r^{2 m-4}\left(r^{4}-2 r^{2} \cos (2 m \theta)+1\right)+\varphi^{\prime 2}, \\
F & =2 r^{2 m-1} \sin (2 m \theta)+a \varphi^{\prime}, \\
G & =r^{2 m-2}\left(r^{4}+2 r^{2} \cos (2 m \theta)+1\right)+a^{2},
\end{aligned}
$$

$m \in \mathbb{R}-\{-1,1\}, r \in \mathbb{R}^{+}, \theta \in I \subset \mathbb{R}$, and the pitch $a \in \mathbb{R}^{+}$.

Proof. The line element of the the helicoidal surface $\mathbf{H}_{m}(r, \theta)$ is

$$
\begin{aligned}
d s^{2}= & {\left[r^{2 m-4}\left(r^{4}-2 r^{2} \cos (2 m \theta)+1\right)+\varphi^{\prime 2}\right] d r^{2} } \\
& +2\left(2 r^{2 m-1} \sin (2 m \theta)+a \varphi^{\prime}\right) d r d \theta \\
& +\left[r^{2 m-2}\left(r^{4}+2 r^{2} \cos (2 m \theta)+1\right)+a^{2}\right] d \theta^{2} .
\end{aligned}
$$

Helices in $\mathbf{H}_{m}(r, \theta)$ are curves defined by $r=$ const. So curves in $\mathbf{H}_{m}(r, \theta)$ that are orthogonal to helices supply the orthogonality condition $F d r+G d \theta=0$. Thus, we 
obtain $\theta=-\int \frac{F}{G} d r+c$, where $c$ is constant. Hence if we put $\bar{\theta}=\theta+\int \frac{F}{G} d r$, then curves orthogonal to helices are given by $\bar{\theta}=$ const.. Substituting the equation $d \theta=d \bar{\theta}-\frac{F}{G} d r$ into the line element (3.4), we have

$$
d s^{2}=\frac{Q}{G} d r^{2}+G d \bar{\theta}^{2}
$$

where $Q:=\operatorname{det} I$. Setting $\bar{r}:=\int \sqrt{\frac{Q}{G}} d r, k(\bar{r}):=\sqrt{G},(3.5)$ becomes

$$
d s^{2}=d \bar{r}^{2}+k^{2}(\bar{r}) d \bar{\theta}^{2} .
$$

The rotational surface

$$
\mathbf{R}_{m}\left(r_{\mathbf{R}}, \theta_{\mathbf{R}}\right)=\left(\begin{array}{c}
\frac{r_{\mathbf{R}}^{m-1}}{m-1} \cos \left[(m-1) \theta_{\mathbf{R}}\right]-\frac{r_{\mathbf{R}}^{m+1}}{m+1} \cos \left[(m+1) \theta_{\mathbf{R}}\right] \\
-\frac{r_{\mathbf{R}}^{m-1}}{m-1} \sin \left[(m-1) \theta_{\mathbf{R}}\right]-\frac{r_{\mathbf{R}}^{m+1}}{m+1} \sin \left[(m+1) \theta_{\mathbf{R}}\right] \\
\varphi_{\mathbf{R}}\left(r_{\mathbf{R}}\right)
\end{array}\right)
$$

has the line element

$$
d s_{\mathbf{R}}^{2}=\frac{Q_{\mathbf{R}}}{G_{\mathbf{R}}} d r_{\mathbf{R}}^{2}+G_{\mathbf{R}} d \bar{\theta}_{\mathbf{R}}^{2}
$$

where

$$
\begin{aligned}
E_{\mathbf{R}} & =r_{\mathbf{R}}^{2 m-4}\left(r_{\mathbf{R}}^{4}-2 r_{\mathbf{R}}^{2} \cos \left(2 m \theta_{\mathbf{R}}\right)+1\right)+\varphi_{\mathbf{R}}^{\prime 2}, \\
F_{\mathbf{R}} & =2 r_{\mathbf{R}}^{2 m-1} \sin \left(2 m \theta_{\mathbf{R}}\right), \\
G_{\mathbf{R}} & =r_{\mathbf{R}}^{2 m-2}\left(r_{\mathbf{R}}^{4}+2 r_{\mathbf{R}}^{2} \cos \left(2 m \theta_{\mathbf{R}}\right)+1\right) .
\end{aligned}
$$

Again, setting $\bar{r}_{\mathbf{R}}:=\int \sqrt{\frac{Q_{\mathbf{R}}}{G_{\mathbf{R}}}} d r_{\mathbf{R}}, \quad k_{\mathbf{R}}\left(\bar{r}_{\mathbf{R}}\right):=\sqrt{G_{\mathbf{R}}}$, then (3.8) becomes

$$
d s_{\mathbf{R}}^{2}=d \bar{r}_{\mathbf{R}}^{2}+k_{\mathbf{R}}^{2}\left(\bar{r}_{\mathbf{R}}\right) d \bar{\theta}_{\mathbf{R}}^{2} .
$$

Comparing (3.6) with (3.9), if we take $\bar{r}=\bar{r}_{\mathbf{R}}, \bar{\theta}=\bar{\theta}_{\mathbf{R}}, k(\bar{r})=k_{\mathbf{R}}\left(\bar{r}_{\mathbf{R}}\right)$, then we have an isometry between $\mathbf{H}_{m}(r, \theta)$ and $\mathbf{R}_{m}\left(r_{\mathbf{R}}, \theta_{\mathbf{R}}\right)$. Therefore, it follows that

$$
\int \sqrt{\frac{Q}{G}} d r=\int \sqrt{\frac{Q_{\mathbf{R}}}{G_{\mathbf{R}}}} d r_{\mathbf{R}}
$$

Substituting the equation

$$
d r_{\mathbf{R}}=\frac{2(m+1) r^{2 m+1}+4 m r^{2 m-1} \cos (2 m \theta)}{2(m+1) r_{\mathbf{R}}^{2 m+1}+4 m r_{\mathbf{R}}^{2 m-1} \cos \left(2 m \theta_{\mathbf{R}}\right)} d r
$$

into the (3.10), we get the function $\varphi_{\mathbf{R}}$.

\section{Helicoidal surface of value 3}

We give the helicoidal surface of value 3 using Bour's theorem in this section.

Proposition 4.1. A helicoidal surface of value 3 (see Figure 1 a-b)

$$
\mathbf{H}_{3}(r, \theta)=\left(\begin{array}{c}
\frac{r^{2}}{2} \cos (2 \theta)-\frac{r^{4}}{4} \cos (4 \theta) \\
-\frac{r^{2}}{2} \sin (2 \theta)-\frac{r^{4}}{4} \sin (4 \theta) \\
\varphi(r)+a \theta
\end{array}\right),
$$


is isometric to the rotational surface of value 3

$$
\mathbf{R}_{3}\left(r_{\mathbf{R}}, \theta_{\mathbf{R}}\right)=\left(\begin{array}{c}
\frac{G}{2} \cos \left[2\left(\theta+\int \frac{F}{G} d r\right)\right]-\frac{G^{2}}{4} \cos \left[4\left(\theta+\int \frac{F}{G} d r\right)\right] \\
-\frac{G}{2} \sin \left[2\left(\theta+\int \frac{F}{G} d r\right)\right]-\frac{G^{2}}{4} \sin \left[4\left(\theta+\int \frac{F}{G} d r\right)\right] \\
\varphi_{\mathbf{R}}\left(r_{\mathbf{R}}\right)
\end{array}\right)
$$

where

$$
\begin{aligned}
\varphi_{\mathbf{R}}^{\prime 2}= & \frac{\left\{8 G^{7 / 2}+12 G^{5 / 2} \cos \left[6\left(\theta+\int \frac{F}{G} d r\right)\right]\right\}^{2} \operatorname{det} I}{\left[8 r^{7}+12 r^{5} \cos (6 \theta)\right]^{2} G} \\
& +\frac{2 G^{3} \sin ^{2}\left[6\left(\theta+\int \frac{F}{G} d r\right)\right]}{G^{2}+2 G \cos \left[6\left(\theta+\int \frac{F}{G} d r\right)\right]+1} \\
& -G\left\{G^{2}-2 G \cos \left[6\left(\theta+\int \frac{F}{G} d r\right)\right]+1\right\}, \\
E & =r^{2}\left[r^{4}-2 r^{2} \cos (6 \theta)+1\right]+\varphi^{\prime 2}, \\
F & =2 r^{5} \sin (6 \theta)+a \varphi^{\prime} \\
G & =r^{4}\left[r^{4}+2 r^{2} \cos (6 \theta)+1\right]+a^{2},
\end{aligned}
$$

$\operatorname{det} I=E G-F^{2}, r, a \in \mathbb{R}^{+}, 0 \leq \theta \leq 2 \pi$.

Proof. Taking $m=3$ in the previous theorem, we easily get the results.

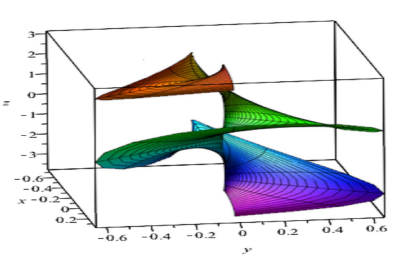

(a)

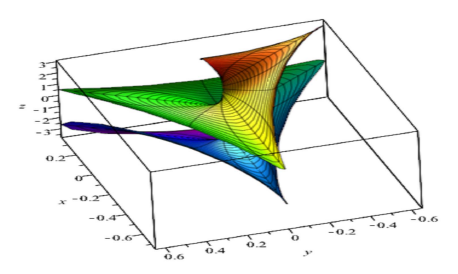

(b)

Figure 1. Helicoidal surface of value $3, \varphi(r)=\frac{2}{3} r^{3} \cos (3 \theta)$.

Corollary 4.1. When $a=0$ and $\varphi(r)=\frac{2}{3} r^{3} \cos (3 \theta)$ in $\mathbf{H}_{3}(r, \theta)$, we obtain the Bour's minimal surface $\mathfrak{B}_{3}(r, \theta)$ (see Figure $2 a-b$, and [10] for details). 


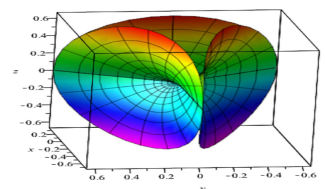

(a)

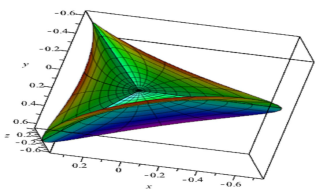

(b)

Figure 2. Bour's minimal surface of value $3, a=0, \varphi(r)=\frac{2}{3} r^{3} \cos (3 \theta)$.

Proposition 4.2. The mean curvature and the Gaussian curvature of the helicoidal surface of value 3 are as follow

$$
\begin{aligned}
H= & \frac{1}{4(\operatorname{det} I)^{3 / 2}}\left\{2 r^{3}\left(r^{4}-1\right)\left(r^{8}+2 r^{6} \cos (6 \theta)+r^{4}+a^{2}\right) \varphi^{\prime \prime}\right. \\
& +4 r^{4}\left(2 r^{4}+r^{2} \cos 6 \theta-1\right) \varphi^{\prime 3}-12 a r^{5} \sin (6 \theta) \varphi^{\prime 2} \\
& +r^{2}\left[2\left(r^{2}+1\right)\left(r^{8}+r^{4}+a^{2}\right) \cos (2 \theta)+\left(2 r^{2}-1\right)\left(r^{8}+r^{4}+a^{2}\right) \cos (4 \theta)\right. \\
& -2 r^{2}\left(10 r^{8}+6 r^{6} \cos (6 \theta)-6 r^{4}+5 a^{2}\right) \cos (6 \theta) \\
& +3 r^{4}\left(r^{8}+r^{4}+a^{2}\right) \cos (8 \theta)-16 r^{8} \sin ^{2} 6 \theta \\
& +4 r^{6}\left(r^{2}+1\right) \cos (2 \theta) \cos (6 \theta)+2 r^{6}\left(2 r^{2}-1\right) \cos (4 \theta) \cos (6 \theta) \\
& \left.+6 r^{10} \cos 6 \theta \cos 8 \theta+r^{4}\left(13 a^{2}+2 r^{4}+5 r^{8}-3\right)-7 a^{2}\right] \varphi^{\prime} \\
& +2\left(2 a r^{3}+1\right)\left(r^{8}+r^{4}+a^{2}\right) \sin (2 \theta)+r(a+2 r)\left(r^{8}+r^{4}+a^{2}\right) \sin (4 \theta) \\
& +2 a r^{3}\left(15 r^{8}-9 r^{4}+a^{2}\right) \sin (6 \theta)-3 a r^{5}\left(r^{8}+r^{4}+a^{2}\right) \sin (8 \theta) \\
& +4 r^{6}\left(2 a r^{3}+1\right) \sin (2 \theta) \cos (6 \theta)+2 r^{7}(a+2 r) \sin (4 \theta) \cos (6 \theta) \\
& \left.-2 a r^{9}\left(2 \sin (6 \theta)+3 r^{2} \sin (8 \theta)\right) \cos (6 \theta)\right\}
\end{aligned}
$$

and

$$
\begin{aligned}
K= & \frac{1}{(\operatorname{det} I)^{2}}\left\{2 a r^{5}\left(r^{4}-1\right)\left(r^{2} \cos (6 \theta)-2 r^{4}+1\right) \varphi^{\prime \prime}+2 r^{8}\left(r^{4}-1\right) \sin (6 \theta) \varphi^{\prime} \varphi^{\prime \prime}\right. \\
& +\frac{1}{2} r^{7}\left[-32 r^{9}+28 r^{5}-8 r+\left(-3 r^{4}+2 r^{2}-1\right) \sin (2 \theta)+2\left(r^{2}+1\right) \sin (4 \theta)\right. \\
& +2\left(-3 r^{4}+1\right) \sin (6 \theta)+2\left(r^{2}+1\right) \sin (8 \theta)+\left(2 r^{2}-1\right) \sin (10 \theta)-2 r^{2} \sin (12 \theta) \\
& \left.+3 r^{4} \sin (14 \theta)+16 r^{3}\left(-2 r^{4}+1\right) \cos (6 \theta)-4 r^{5} \cos (12 \theta)\right] \varphi^{\prime 2} \\
& +r^{4}\left[8 a r^{5}\left(2 r^{4}-1\right) \sin (6 \theta)+4 a r^{7} \sin (12 \theta)+\left(-4 a r^{6}-3 a r^{4}+r^{3}+2 a r^{2}+2 a\right) \cos (2 \theta)\right. \\
& +\left(-4 a r^{6}+5 a r^{4}+3 a r^{2}+r-a\right) \cos (4 \theta)+a\left(r^{4}-1\right) r^{2} \cos (6 \theta) \\
& +\left(-6 a r^{8}+2 a r^{4}+a r^{2}-r\right) \cos (8 \theta)+\left(a r^{4}-r^{3}-a r^{2}\right) \cos (10 \theta) \\
& \left.-2 a r^{4} \cos (12 \theta)+3 a r^{6} \cos (14 \theta)+6 a r^{8}-5 a r^{4}+a\right] \varphi^{\prime} \\
& -\frac{1}{2} a r^{2}\left(19 a r^{7}-7 a r^{3}+10 r^{2}-4\right) \sin (2 \theta)+a r^{2}\left(1-4 r^{4}\right)(a r+1) \sin (4 \theta) \\
& -2 a^{2} r^{5}\left(2 r^{4}-1\right) \sin (6 \theta)+a r^{4}\left(6 a r^{7}-a r^{3}+1\right) \sin (8 \theta)+\frac{1}{2} a r^{4}(a r+2) \sin (10 \theta) \\
& \left.+a^{2} r^{7} \sin (12 \theta)-\frac{3}{2} a^{2} r^{9} \sin (14 \theta)+2 a^{2} r^{10} \cos (12 \theta)-2 a^{2} r^{10}\right\} .
\end{aligned}
$$


respectively, where $\operatorname{det} I=E G-F^{2}, \varphi^{\prime}=\frac{d \varphi}{d r}, r, a \in \mathbb{R}^{+}, 0 \leq \theta \leq 2 \pi$.

Proof. Taking the differential with respect to $r, \theta$ to the $\mathbf{H}_{3}$, we have

$$
\left(\mathbf{H}_{3}\right)_{r}=\left(\begin{array}{c}
r \cos (2 \theta)-r^{3} \cos (4 \theta) \\
-r \sin (2 \theta)-r^{3} \sin (4 \theta) \\
\varphi^{\prime}
\end{array}\right)
$$

and

$$
\left(\mathbf{H}_{3}\right)_{\theta}=\left(\begin{array}{c}
-r^{2} \sin (2 \theta)+r^{4} \sin (4 \theta) \\
-r^{2} \cos (2 \theta)-r^{4} \cos (4 \theta) \\
a
\end{array}\right),
$$

The coefficients of the first fundamental form of the surface are

$$
\begin{gathered}
E=r^{2}\left(r^{4}-2 r^{2} \cos (6 \theta)+1\right)+\varphi^{\prime 2}, \\
F=2 r^{5} \sin (6 \theta)+a \varphi^{\prime}, \\
G=r^{4}\left(r^{4}+2 r^{2} \cos (6 \theta)+1\right)+a^{2} .
\end{gathered}
$$

Then we get

$$
\begin{aligned}
\operatorname{det} I= & r^{2}\left[r^{12}-2 r^{8}+a^{2} r^{4}+r^{4}-2 a^{2} r^{2} \cos (6 \theta)+a^{2}\right] \\
& -4 a r^{5} \sin (6 \theta) \varphi^{\prime}+r^{4}\left[r^{4}+2 r^{2} \cos (6 \theta)+1\right] \varphi^{\prime 2} .
\end{aligned}
$$

Using the second differentials

$$
\begin{gathered}
\left(\mathbf{H}_{3}\right)_{r r}=\left(\begin{array}{c}
\cos (2 \theta)-3 r^{2} \cos (4 \theta) \\
-\sin (2 \theta)-3 r^{2} \sin (4 \theta) \\
\varphi^{\prime \prime}
\end{array}\right), \\
\left(\mathbf{H}_{3}\right)_{r \theta}=\left(\begin{array}{c}
-2 r \sin (2 \theta)+4 r^{3} \sin (4 \theta) \\
-2 r \cos (2 \theta)-4 r^{3} \cos (4 \theta) \\
0
\end{array}\right), \\
\left(\mathbf{H}_{3}\right)_{\theta \theta}=\left(\begin{array}{c}
-2 r^{2} \cos (2 \theta)+4 r^{4} \cos (4 \theta) \\
2 r^{2} \sin (2 \theta)+4 r^{4} \sin (4 \theta) \\
0
\end{array}\right),
\end{gathered}
$$

and the Gauss map (the unit normal)

$$
e=\frac{1}{\sqrt{\operatorname{det} I}}\left(\begin{array}{c}
-\operatorname{ar}\left(r^{2} \sin (4 \theta)+\sin (2 \theta)\right)+r^{2} \varphi^{\prime}\left(r^{2} \cos (4 \theta)+\cos (2 \theta)\right) \\
\operatorname{ar}\left(r^{2} \cos (4 \theta)-\cos (2 \theta)\right)+r^{2} \varphi^{\prime}\left(r^{2} \sin (4 \theta)-\sin (2 \theta)\right) \\
r^{7}-r^{3}
\end{array}\right)
$$

of the surface $\mathbf{H}_{3}$, we have the coefficients of the second fundamental form of the surface as follow

$$
\begin{aligned}
L= & \frac{1}{\sqrt{\operatorname{det} I}}\left(r^{3}\left(r^{4}-1\right) \varphi^{\prime \prime}+\frac{1}{2} r^{2}\left(1-3 r^{4}+2\left(1+r^{2}\right) \cos 2 \theta\right.\right. \\
& \left.+\left(-1+2 r^{2}\right) \cos 4 \theta-2 r^{2} \cos 6 \theta+3 r^{4} \cos 8 \theta\right) \varphi^{\prime} \\
& +\left(1+2 a r^{3}\right) \sin 2 \theta+\frac{1}{2} r(a+2 r) \sin 4 \theta \\
& \left.+a r^{3} \sin 6 \theta-\frac{3}{2} a r^{5} \sin 8 \theta\right), \\
M= & \frac{1}{\sqrt{\operatorname{det} I}} 2 r^{2}\left(\left(-2 r^{4}+r^{2} \cos 6 \theta+1\right) a+r^{3} \varphi^{\prime} \sin 6 \theta\right),
\end{aligned}
$$


and

$$
N=\frac{1}{\sqrt{\operatorname{det} I}} 2 r^{4}\left(-\operatorname{ar} \sin 6 \theta+\left(2 r^{4}+r^{2} \cos 6 \theta-1\right) \varphi^{\prime}\right)
$$

Therefore, we can see the results easily.

Corollary 4.2. If the helicoidal surface of value 3 is minimal then we get

$$
\begin{aligned}
0= & 2 r^{3}\left(r^{4}-1\right)\left(r^{8}+2 r^{6} \cos (6 \theta)+r^{4}+a^{2}\right) \varphi^{\prime \prime} \\
& +4 r^{4}\left(2 r^{4}+r^{2} \cos 6 \theta-1\right) \varphi^{\prime 3}-12 a r^{5} \sin (6 \theta) \varphi^{\prime 2} \\
& +r^{2}\left[2\left(r^{2}+1\right)\left(r^{8}+r^{4}+a^{2}\right) \cos (2 \theta)+\left(2 r^{2}-1\right)\left(r^{8}+r^{4}+a^{2}\right) \cos (4 \theta)\right. \\
& -2 r^{2}\left(10 r^{8}+6 r^{6} \cos (6 \theta)-6 r^{4}+5 a^{2}\right) \cos (6 \theta) \\
& +3 r^{4}\left(r^{8}+r^{4}+a^{2}\right) \cos (8 \theta)-16 r^{8} \sin ^{2} 6 \theta \\
& +4 r^{6}\left(r^{2}+1\right) \cos (2 \theta) \cos (6 \theta)+2 r^{6}\left(2 r^{2}-1\right) \cos (4 \theta) \cos (6 \theta) \\
& \left.+6 r^{10} \cos 6 \theta \cos 8 \theta+r^{4}\left(13 a^{2}+2 r^{4}+5 r^{8}-3\right)-7 a^{2}\right] \varphi^{\prime} \\
& +2\left(2 a r^{3}+1\right)\left(r^{8}+r^{4}+a^{2}\right) \sin (2 \theta)+r(a+2 r)\left(r^{8}+r^{4}+a^{2}\right) \sin (4 \theta) \\
& +2 a r^{3}\left(15 r^{8}-9 r^{4}+a^{2}\right) \sin (6 \theta)-3 a r^{5}\left(r^{8}+r^{4}+a^{2}\right) \sin (8 \theta) \\
& +4 r^{6}\left(2 a r^{3}+1\right) \sin (2 \theta) \cos (6 \theta)+2 r^{7}(a+2 r) \sin (4 \theta) \cos (6 \theta) \\
& -2 a r^{9}\left(2 \sin (6 \theta)+3 r^{2} \sin (8 \theta)\right) \cos (6 \theta)
\end{aligned}
$$

The solution of the second order ODE as above is an atractive problem.

The author also focuses on the spacelike and timelike helicoidal surfaces of value $m$ in the Minkowski 3-space $\mathbb{L}^{3}$ in the next papers.

Acknowledgement. This work had been stated by the author, when he visited as a post-doctoral researcher at the Katholieke Universiteit Leuven, Belgium in 2011-2012 academic year. The author would like to thank to the hospitality of the members of the geometry section at K.U. Leuven. Especially to the Professor Franki Dillen, and Dr. Ana Nistor.

\section{REFERENCES}

[1] Baikoussis, Chr., Koufogiorgos, T., Helicoidal surfaces with prescribed mean or Gaussian curvature, J. Geom. 63 (1998) 25-29.

[2] Beneki, Chr. C., Kaimakamis, G., Papantoniou, B.J., A classification of surfaces of revolution of constant Gaussian curvature in the Minkowski space $R_{1}^{3}$, Bull. Calcutta Math. Soc. 90 (1998) 441-458.

[3] Beneki, Chr. C., Kaimakamis, G., Papantoniou, B.J., Helicoidal surfaces in three-dimensional Minkowski space, J. Math. Anal. Appl., 275 (2002) 586-614.

[4] Bour, E., Théorie de la déformation des surfaces. J. de l'Êcole Imperiale Polytechnique, 22-39 (1862) 1-148.

[5] Do Carmo, M., Dajczer, M., Helicoidal surfaces with constant mean curvature, Tohôku Math. J. 34 (1982) 351-367.

[6] Dillen, F., Kühnel, W., Ruled Weingarten surfaces in Minkowski 3-space, Manuscripta Math. 98 (1999) 307-320.

[7] Eisenhart, L., A Treatise on the Differential Geometry of Curves and Surfaces, Palermo 41 Ginn and Company, 1909.

[8] Güler, E., Bour's theorem and lightlike profile curve. Yokohama Math. J., 54-1 (2007) 55-77.

[9] Güler, E., Yaylı, Y., Hacısalihoğlu, H.H., Bour's theorem on Gauss map in Euclidean 3-space, Hacettepe J. Math. Stat. 39-4 (2010) 515-525. 
[10] Güler, E., Bour's minimal surface in three dimensional Lorentz-Minkowski space, (presented in GeLoSP2013, VII International Meetings on Lorentzian Geometry, Sao Paulo University, Sao Paulo, Brasil) preprint.

[11] Hitt, L, Roussos, I., Computer graphics of helicoidal surfaces with constant mean curvature, An. Acad. Brasil. Ciênc. 63 (1991) 211-228.

[12] Ikawa, T., Bour's theorem and Gauss map, Yokohama Math. J. 48-2 (2000) 173-180.

[13] Ikawa, T., Bour's theorem in Minkowski geometry, Tokyo J.Math. 24 (2001) 377-394.

[14] Kenmotsu, K., Surfaces of revolution with prescribed mean curvature, Tohôku Math. J. 32 (1980) 147-153.

[15] Spivac, M., A Comprehensive Introduction to Differential Geometry III, Interscience, New York, 1969.

[16] Struik, D.J., Lectures on Differential Geometry, Addison-Wesley, 1961.

Bartin University, Faculty of Science, Department of Mathematics, 74100, Bartin, TURKEY.

E-mail address: ergler@gmail.com 